\title{
Bicoprime Factor Robust Control Synthesis via Reduced Dimension Algebraic Riccati Equations
}

DOI:

10.23919/ECC.2019.8796183

\section{Document Version}

Accepted author manuscript

Link to publication record in Manchester Research Explorer

\section{Citation for published version (APA):}

Tsiakkas, M., \& Lanzon, A. (2019). Bicoprime Factor Robust Control Synthesis via Reduced Dimension Algebraic Riccati Equations. In Proceedings of the 2019 European Control Conference

https://doi.org/10.23919/ECC.2019.8796183

\section{Published in:}

Proceedings of the 2019 European Control Conference

\section{Citing this paper}

Please note that where the full-text provided on Manchester Research Explorer is the Author Accepted Manuscript or Proof version this may differ from the final Published version. If citing, it is advised that you check and use the publisher's definitive version.

\section{General rights}

Copyright and moral rights for the publications made accessible in the Research Explorer are retained by the authors and/or other copyright owners and it is a condition of accessing publications that users recognise and abide by the legal requirements associated with these rights.

\section{Takedown policy}

If you believe that this document breaches copyright please refer to the University of Manchester's Takedown Procedures [http://man.ac.uk/04Y6Bo] or contact uml.scholarlycommunications@manchester.ac.uk providing relevant details, so we can investigate your claim.

\section{OPEN ACCESS}




\title{
Bicoprime Factor Robust Control Synthesis via Reduced Dimension Algebraic Riccati Equations
}

\author{
Mihalis Tsiakkas $^{1, *}$ and Alexander Lanzon ${ }^{2}$
}

\begin{abstract}
Bicoprime factorizations of the plant have recently resurfaced in control theory, after being a largely dormant subject for three decades. Recent results have shown that bicoprime factorizations can be beneficial in solving some control problems. In this paper, an additional advantage is presented where the use of bicoprime factorizations of the plant allow for robust control synthesis via reduced dimension algebraic Riccati equations by discarding some (or all) of the stable dynamics of the plant. This is a major advantage in the stabilisation of high order systems for which robust control synthesis can become computationally intractable.
\end{abstract}

\section{INTRODUCTION}

Bicoprime factorizations (BCFs) of the plant over $\mathscr{R} \mathscr{H}_{\infty}$ were recently reintroduced to the control community in [1], [2]. Though these factorizations were proposed more than three decades ago, in fact BCFs are derivative of the polynomial methods developed by Rosenbrock [3], however little was done to advance the field. The primary reason for this seems to be the fact that coprime factorizations and state space methods (which BCFs generalize) are very easy to work with and produce powerful results. It was shown in [1], [2] that using BCFs can yield computational advantages in internal stability analysis.

BCFs received brief attention in the past and were shown to be good candidates in the development of decentralized control strategies. For example, a Youla type controller parametrisation was presented in [4] utilising BCFs; these results were then extended and put in a decentralized control context by [5]. Furthermore, in [6] BCFs were used to enumerate the so-called decentralized blocking zeros of a system which assist in deducing the existence of a decentralized stabilising controller.

Coprime factorizations (which form a special subset of BCFs) have found extended applications in control theory [7]. Coprime factor uncertainty was first proposed by [8]. It was argued therein, and corroborated by many other authors that such an uncertainty structure is superior to others (such as additive, multiplicative, etc.). Some of the appealing properties of coprime factor uncertainty include the ability to model both the number and location of uncertain right half plane poles while in the normalized case the resulting stability margin can be shown to be equivalent to the one

\footnotetext{
${ }^{1}$ Mihalis Tsiakkas is with the KIOS Research and Innovation Center of Excellence, University of Cyprus, Nicosia, Cyprus. m.tsiakkas@ieee.org

${ }^{2}$ Alexander Lanzon is with the Control Systems Centre, School of Electrical and Electronic Engineering, University of Manchester, Manchester, M13 9PL, UK a. lanzon@ieee.org

* Corresponding author.
}

obtained from the four block problem. BCF uncertainty was first proposed in [1], [2], where it was shown to encompass and generalize the coprime factor uncertainty structure. For a brief comparison of the two, the reader is referred to [2].

In this paper, it is shown that employing a strategically constructed $\mathrm{BCF}$ of the plant can lead to computational advantages in robust control synthesis allowing for the synthesis of a robustly stabilizing controller using the formulation of [9] via the solution of two reduced dimension Algebraic Riccati Equations (AREs). The proposed procedure provides a method to discard some or even all of the plant's stable modes during $\mathscr{H}_{\infty}$ robust control synthesis without imposing or assuming uncontrollability or unobservability. This can be beneficial for very high order systems with numerous open left half plane poles where solving the standard AREs associated with $\mathscr{H}_{\infty}$ synthesis can present numerical difficulties.

\section{PRELIMINARIES}

The sets $\mathbb{R}$ and $\mathbb{C}$ are defined as the real and complex numbers respectively. Let $A \in \mathbb{C}^{m \times n}$ then $A^{*}$ denotes its complex conjugate transpose of $A$. The rank of $A$ is denoted by rank $A$. If $m=n, \Lambda(A)$ is the spectrum of $A$ and $\rho(A)$ is its spectral radius; furthermore, the notation $A>0$ (resp. $A \geq 0$ ) is used to denote that $A$ is positive definite (resp. semi-definite). Given two matrices $A \in \mathbb{R}^{m \times n}$ and $B \in$ $\mathbb{R}^{p \times q}, A \otimes B \in \mathbb{R}^{m p \times n q}$ denotes the Kronecker product of the two.

The set of all real, rational, proper transfer matrices is denoted $\mathscr{R}$. The subset of $\mathscr{R}$ containing all stable transfer matrices is given by $\mathscr{R} \mathscr{H}$.

Let $H \in \mathscr{R}$ and $\Delta \in \mathscr{R}$, then the lower and upper linear fractional transformations (LFTs) of $H$ with respect to $\Delta$ are given by $\mathcal{F}_{l}(H, \Delta)$ and $\mathcal{F}_{u}(H, \Delta)$ respectively. See [10, Chapter 10] for further details.

Let $P \in \mathscr{R}$, then $P=\left[\begin{array}{l|l}A & B \\ \hline C & D\end{array}\right]$ is shorthand for $P=$ $C(s I-A)^{-1} B+D$.

The notation adopted herein with regards to AREs is that of [10, Chapter 13]. Specifically, Ric is defined as the Riccati operator mapping a Hamiltonian matrix to the stabilizing solution of the associated ARE (if it exists) and dom(Ric) the domain of Ric.

Left and right coprime factorizations (LCF and RCF respectively) are invaluable tools in control theory with uses ranging from control synthesis [11] to distance measures [12]. A pair $\{L, M\}$ is said to be left coprime (LC) over $\mathscr{R} \mathscr{H}_{\infty}$ if $L, M \in \mathscr{R} \mathscr{H}_{\infty}$ and there exist $X, Y \in \mathscr{R} \mathscr{H}_{\infty}$ 
such that $M X+L Y=I$. Furthermore, the pair is a LCF of a plant $P \in \mathscr{R}$ if it is LC, $M$ is square with $\operatorname{det} M(\infty) \neq 0$ and $P=M^{-1} L$. Similarly, the pair $\{N, M\}$ is right coprime (RC) over $\mathscr{R} \mathscr{H}_{\infty}$ if $N, M \in \mathscr{R} \mathscr{H}_{\infty}$ and there exist $X, Y \in \mathscr{R} \mathscr{H}_{\infty}$ such that $X M+Y N=I$. A RCF is defined dually to a LCF with the pair being a RCF of a plant $P \in \mathscr{R}$ if it is RC, $M$ is square with $\operatorname{det} M(\infty) \neq 0$ and $P=N M^{-1}$.

The following lemma outlines a special case of an ARE where the solution can be obtained by solving a reduced order equation corresponding to a subset of the ARE. This forms the basis of the main result of the present paper.

Lemma 1: Consider the continuous time ARE

$$
A^{*} X+X A+X S X+C^{*} C=0
$$

where $A=\left[\begin{array}{cc}A_{+} & 0 \\ 0 & A_{-}\end{array}\right] \in \mathbb{R}^{n \times n}$ with $A_{+} \in \mathbb{R}^{n_{+} \times n_{+}}$and $A_{-}$ Hurwitz, $S=\left[\begin{array}{ll}S_{11} & S_{12} \\ S_{12}^{*} & S_{22}\end{array}\right] \in \mathbb{R}^{n \times n}$ with $S_{11} \in \mathbb{R}^{n_{+} \times n_{+}}$and $C=\left[\begin{array}{ll}C_{1} & 0\end{array}\right] \in \mathbb{R}^{p \times n}$ with $C_{1} \in \mathbb{R}^{p \times n_{+}}$. Then the stabilizing solution of (1) is given by $X=\left[\begin{array}{cc}X^{\prime} & 0 \\ 0 & 0\end{array}\right]$ where $X^{\prime} \in \mathbb{R}^{n_{+} \times n_{+}}$ is the stabilizing solution to the reduced dimension ARE

$$
A_{+}^{*} X^{\prime}+X^{\prime} A_{+}+X^{\prime} S_{11} X^{\prime}+C_{1}^{*} C_{1}=0 .
$$

Proof: By definition $X$ is a stabilizing solution if and only if $A+S X$ is Hurwitz. Let $X^{\prime}$ be the stabilising solution to (2) and define $X=\left[\begin{array}{cc}X^{\prime} & 0 \\ 0 & 0\end{array}\right]$. Then

$$
\begin{aligned}
A+S X & =\left[\begin{array}{cc}
A_{+} & 0 \\
0 & A_{-}
\end{array}\right]+\left[\begin{array}{ll}
S_{11} & S_{12} \\
S_{12}^{*} & S_{22}
\end{array}\right]\left[\begin{array}{cc}
X^{\prime} & 0 \\
0 & 0
\end{array}\right] \\
& =\left[\begin{array}{cc}
A_{+}+S_{11} X^{\prime} & 0 \\
S_{12}^{*} X^{\prime} & A_{-}
\end{array}\right] .
\end{aligned}
$$

Then $A+S X$ is Hurwitz since both $A_{-}$and $A_{+}+S_{11} X^{\prime}$ are Hurwitz. Proving that $X$ also satisfies (1) is trivial and thus omitted.

\section{BCF PRELIMINARIES}

BCFs first appeared in the literature in [7] where their existence was acknowledged with no significant results given. In the original definition, BCFs of a plant were presented as a quad of objects in $\mathscr{R} \mathscr{H}_{\infty}$; this definition follows.

Definition 1 ([7]): The ordered quad $\{N, M, L, K\}$ is bicoprime (BC) in $\mathscr{R} \mathscr{H}_{\infty}$ if $\{L, M\}$ is LC in $\mathscr{R} \mathscr{H}_{\infty},\{N, M\}$ is $\mathrm{RC}$ in $\mathscr{R} \mathscr{H}_{\infty}$ and $K \in \mathscr{R} \mathscr{H}_{\infty}$. Furthermore, the quad is a BCF of a plant $P \in \mathscr{R}$ over $\mathscr{R} \mathscr{H}_{\infty}$ if it is BC, $M$ is square, $\operatorname{det} M(\infty) \neq 0$ and $P=N M^{-1} L+K$.

The following definition presents the notation used for the sets of all BC quads and BCFs of a plant.

Definition 2: The set of all BC quads in $\mathscr{R} \mathscr{H}_{\infty}$ is denoted by $\mathscr{B}$, while the set of all BCFs of a plant $P \in \mathscr{R}$ over $\mathscr{R} \mathscr{H}_{\infty}$ is denoted by $\mathscr{B}(P)$.

It is often convenient to pack a $\mathrm{BC}$ quad into a matrix as in the following definition.

Definition 3: The set $\tilde{\mathscr{B}}$ is defined as

$$
\tilde{\mathscr{B}}=\left\{\left[\begin{array}{cc}
M & -L \\
N & K
\end{array}\right]:\{N, M, L, K\} \in \mathscr{B}\right\} .
$$

When representing a $\mathrm{BCF}$ of a plant $P \in \mathscr{R}$, the notation $\tilde{\mathscr{B}}(P)$ will be used.
Formulae for computing L/RCFs of plant using state space data were first given by [13]. The following theorem presents a similar result for constructing a $\mathrm{BCF}$ of a plant.

Theorem 1 ([2]): Let $P \in \mathscr{R}^{p \times q}$ have a stabilizable and detectable state space realization $P=\left[\begin{array}{l|l}A & B \\ \hline C & D\end{array}\right]$. Furthermore, suppose that $Q \in \mathbb{R}^{n \times r}$ and $R \in \mathbb{R}^{r \times n}$ are such that $A+Q R$ is Hurwitz. Finally, define

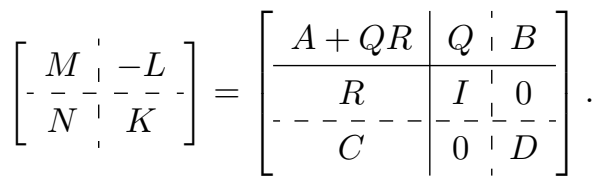

Then $\{N, M, L, K\} \in \mathscr{B}(P)$.

The BCF parametrization presented in Theorem 1 will henceforth be referred to as a $Q R$-BCF parametrisation, as it is purely parametrised by the matrices $Q$ and $R$.

Following coprime factor convention, BCF uncertainty is defined by additive perturbations on the $\mathrm{BC}$ factors. With the resulting perturbed plant given by

$$
P_{\Delta}=\left(N+\Delta_{N}\right)\left(M+\Delta_{M}\right)^{-1}\left(L+\Delta_{L}\right)+\left(K+\Delta_{K}\right) .
$$

In the case of LCF and RCF uncertainty, the perturbed plant is admissible only if coprimeness of the factors is preserved [14, Remark 4.4]. A similar condition, namely that bicoprimeness is preserved under the perturbations of the factors, is imposed herein.

Figure 1 shows a block diagram representation of the proposed BCF uncertainty structure given by (4). It can be observed that this generalizes many of the uncertainty structures studied in the past, for example by [15]. From this a generalised plant and uncertainty matrix can be defined as

$$
\begin{gathered}
\Pi=\left[\begin{array}{cc:c}
M^{-1} & 0 & M^{-1} L \\
0 & 0 & I \\
\hdashline N M^{-1} & I & P
\end{array}\right] \text { and } \\
\Delta=\left[\begin{array}{c:c}
-\Delta_{M} & \Delta_{L} \\
\Delta_{N} & \Delta_{K}
\end{array}\right] .
\end{gathered}
$$

It is straightforward to confirm that using the above $\Pi$ and $\Delta$ yields $P_{\Delta}=\mathcal{F}_{u}(\Pi, \Delta)$.

Let $P \in \mathscr{R}$ have a stabilizable and detectable state space realization $P=\left[\begin{array}{l|l}A & B \\ \hline C & D\end{array}\right]$. Using the $Q R$-BCF parametrisation in (3), the BCF generalized plant $\Pi$ given by (5) can be expressed in state space form as

$$
\Pi=\left[\begin{array}{c:c}
\Pi_{11} & \Pi_{12} \\
\hdashline \Pi_{21} & \bar{\Pi}_{22}
\end{array}\right]=\left[\begin{array}{c|cc:c}
A & Q & 0 & B \\
\hdashline-R & I & 0 & 0 \\
0 & 0 & 0 & I \\
\hdashline C & 0 & I & D
\end{array}\right] .
$$

Using the $Q R$-BCF parametrisation, a BCF $\mathscr{H}_{\infty}$ control synthesis theorem is developed based on the 2-ARE solution to the $\mathscr{H}_{\infty}$ problem given by [9]. This result is given in Theorem 2. 


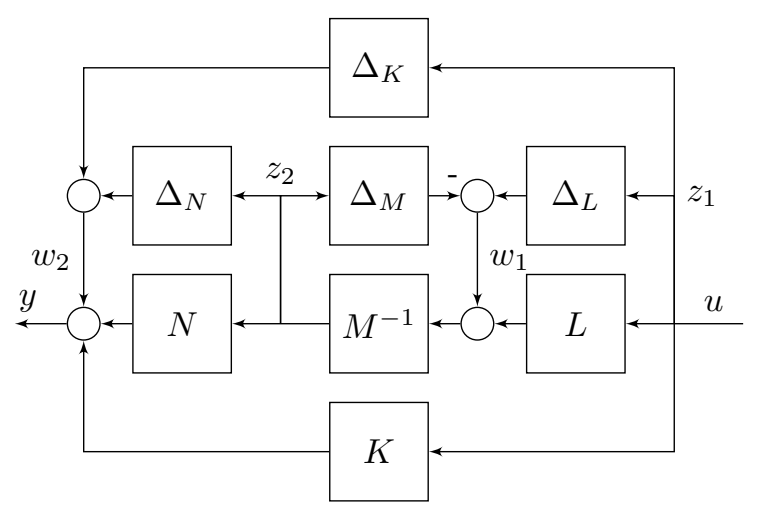

Fig. 1: Perturbed plant block diagram with BC factor uncertainty.

Theorem 2: Let $P \in \mathscr{R}^{p \times q}$ have a stabilizable and detectable state space representation $P=\left[\begin{array}{l|l}A & B \\ \hline C & D\end{array}\right]$ where $D=0^{1}$ and a $Q R$-BCF as outlined in (3). Furthermore, let $0<\gamma \in \mathbb{R}$ and define $\Pi$ as in (7),

$$
H_{\infty}=\left[\begin{array}{cc}
A-\frac{1}{\gamma^{2}-1} Q R & \frac{1}{\gamma^{2}-1} Q Q^{*}-B B^{*} \\
-\frac{\gamma^{2}}{\gamma^{2}-1} R^{*} R & -\left(A-\frac{1}{\gamma^{2}-1} Q R\right)^{*}
\end{array}\right]
$$

and

$$
J_{\infty}=\left[\begin{array}{cc}
\left(A-\frac{1}{\gamma^{2}-1} Q R\right)^{*} & \frac{1}{\gamma^{2}-1} R^{*} R-C^{*} C \\
-\frac{\gamma^{2}}{\gamma^{2}-1} Q Q^{*} & -\left(A-\frac{1}{\gamma^{2}-1} Q R\right)
\end{array}\right] .
$$

Then there exists a controller $C_{\infty} \in \mathscr{R}^{q \times p}$ satisfying $\left\|\mathcal{F}_{l}\left(\Pi, C_{\infty}\right)\right\|_{\infty}<\gamma$ if and only if

1) $\gamma>1$;

2) $H_{\infty} \in \operatorname{dom}(\operatorname{Ric})$ and $X_{\infty}=\operatorname{Ric}\left(H_{\infty}\right) \geq 0$;

3) $J_{\infty} \in \operatorname{dom}(\operatorname{Ric})$ and $Y_{\infty}=\operatorname{Ric}\left(J_{\infty}\right) \geq 0$;

4) $\rho\left(X_{\infty} Y_{\infty}\right)<\gamma^{2}$.

If the above conditions hold, the set of all such controllers is given by

$$
\left\{C_{\infty}=\mathcal{F}_{l}\left(\Pi_{\infty}, \Phi\right): \Phi \in \mathscr{R} \mathscr{H}_{\infty},\|\Phi\|_{\infty}<\gamma\right\}
$$

where

$$
\begin{gathered}
\Pi_{\infty}=\left[\begin{array}{c|cc}
A_{\infty} & -Z_{\infty} Y_{\infty} C^{*} & Z_{\infty} B \\
\hline B^{*} X_{\infty} & 0 & I \\
-C & I & 0
\end{array}\right], \\
Z_{\infty}=\left(I-\gamma^{-2} Y_{\infty} X_{\infty}\right)^{-1}, \text { and } \\
A_{\infty}=A-\frac{1}{\gamma^{2}-1} Q R-Z_{\infty} Y_{\infty} C^{*} C \\
\quad+\left(\frac{1}{\gamma^{2}-1} Q Q^{*}-B B^{*}\right) X_{\infty}
\end{gathered}
$$

Proof: The proof follows by direct application of $[9$, Theoresm $3 \& 4]$ to the generalized plant $\Pi$ in (7).

\footnotetext{
${ }^{1}$ The assumption that $P(\infty)=0$ is standard in this context and can be guaranteed via simple loop transformations [10].
}

\section{MAIN RESUlt}

In this section the main result of this paper restricts the synthesis procedure outlined by Theorem 2 to a special class of state space realizations to achieve robust control synthesis via the solution of reduced dimension AREs.

Due to the structure of the $Q R$-BCF parametrisation, it is possible to synthesize a controller by solving reduced dimension AREs which is the main contribution of this paper. Suppose that a plant $P \in \mathscr{R}$ has the stabilizable and detectable state space realization

$$
P=\left[\begin{array}{c|c}
A & B \\
\hline C & D
\end{array}\right]=\left[\begin{array}{cc|c}
A_{+} & 0 & B_{+} \\
0 & A_{-} & B_{-} \\
\hline C_{+} & C_{-} & D
\end{array}\right],
$$

where $A_{-} \in \mathbb{R}^{n_{-} \times n_{-}}$is Hurwitz and $A_{+} \in \mathbb{R}^{n_{+} \times n_{+}}$. Such a realization always exists and is equivalent to decomposing the plant into a stable and anti-stable part. This is often referred to as a Gilbert realization and can be obtained via a Jordan decomposition of $A$.

Now let $Q_{+} \in \mathbb{R}^{n_{+} \times r}$ and $R_{+} \in \mathbb{R}^{r \times n_{+}}$be such that $A_{+}+Q_{+} R_{+}$is Hurwitz. Then a $Q R$-BCF of $P$ is given by

$$
\left[\begin{array}{cc|c:c}
A_{+}+Q_{+} R_{+} & 0 & Q_{+} & B_{+} \\
0 & A_{-} & 0 & B_{-} \\
\hdashline R_{+} & 0 & I & 0 \\
\hdashline C_{+} & C_{-} & 0 & D
\end{array}\right] \in \tilde{\mathscr{B}}(P)
$$

Note that in the above $Q R$-BCF, the modes contained in $A_{-}$ are both controllable and observable only in the additive term of the factorisation.

A new generalized plant $\Pi^{\prime}$ can then be constructed for the uncertainty structure in Figure 1 using the $Q R$-BCF of $P$ given in (11) as follows:

$$
\Pi^{\prime}=\left[\begin{array}{cc|cc:c}
A_{+} & 0 & Q_{+} & 0 & B_{+} \\
0 & A_{-} & 0 & 0 & B_{-} \\
\hline-R_{+} & 0 & I & 0 & 0 \\
0 & 0 & 0 & 0 & I \\
\hdashline C_{+} & C_{-} & 0 & I & D
\end{array}\right] .
$$

Now using $\Pi^{\prime}$ in Theorem 2 results in Hamiltonians where the associated AREs have the structure assumed in Lemma 1. Therefore it is only necessary to solve AREs corresponding to $A_{+}$whose dimension is dictated by $n_{+}$. This fact is formally stated in the following result.

Theorem 3: Let $P \in \mathscr{R}^{p \times q}$ have a stabilizable and detectable state space realization of the form given by (10) with $A_{-}$Hurwitz, $D=0$ and a $Q R$-BCF of the form given by (11). Furthermore, let $0<\gamma \in \mathbb{R}$ and define $\Pi^{\prime}$ as in (12),

$$
H_{\infty}^{\prime}=\left[\begin{array}{cc}
A_{+}-\frac{1}{\gamma^{2}-1} Q_{+} R_{+} & \frac{1}{\gamma^{2}-1} Q_{+} Q_{+}^{*}-B_{+} B_{+}^{*} \\
-\frac{\gamma^{2}}{\gamma^{2}-1} R_{+}^{*} R_{+} & -\left(A_{+}-\frac{1}{\gamma^{2}-1} Q_{+} R_{+}\right)^{*}
\end{array}\right]
$$


and

$$
J_{\infty}^{\prime}=\left[\begin{array}{cc}
\left(A_{+}-\frac{1}{\gamma^{2}-1} Q_{+} R_{+}\right)^{*} & \frac{1}{\gamma^{2}-1} R_{+}^{*} R_{+}-C_{+}^{*} C_{+} \\
-\frac{\gamma^{2}}{\gamma^{2}-1} Q_{+} Q_{+}^{*} & -\left(A_{+}-\frac{1}{\gamma^{2}-1} Q_{+} R_{+}\right)
\end{array}\right] .
$$

Then there exists a controller $C_{\infty}^{\prime} \in \mathscr{R}^{q \times p}$ satisfying $\left\|\mathcal{F}_{l}\left(\Pi^{\prime}, C_{\infty}^{\prime}\right)\right\|_{\infty}<\gamma$ if and only if

1) $\gamma>1$;

2) $H_{\infty}^{\prime} \in \operatorname{dom}($ Ric $)$ and $X_{\infty}^{\prime}=\operatorname{Ric}\left(H_{\infty}^{\prime}\right) \geq 0$;

3) $J_{\infty}^{\prime} \in \operatorname{dom}(\operatorname{Ric})$ and $Y_{\infty}^{\prime}=\operatorname{Ric}\left(J_{\infty}^{\prime}\right) \geq 0$;

4) $\rho\left(X_{\infty}^{\prime} Y_{\infty}^{\prime}\right)<\gamma^{2}$.

If the above conditions hold, the set of all such controllers is given by

$$
\left\{C_{\infty}^{\prime}=\mathcal{F}_{l}\left(\Pi_{\infty}^{\prime}, \Phi\right): \Phi \in \mathscr{R} \mathscr{H}_{\infty},\|\Phi\|_{\infty}<\gamma\right\}
$$

where

$$
\Pi_{\infty}^{\prime}=\left[\begin{array}{cc|cc}
A_{\infty}^{\prime} & -Z_{\infty}^{\prime} Y_{\infty}^{\prime} C_{+}^{*} C_{-} & -Z_{\infty}^{\prime} Y_{\infty}^{\prime} C_{+}^{*} & Z_{\infty}^{\prime} B_{+} \\
-B_{-} B_{+}^{*} X_{\infty}^{\prime} & A_{-} & 0 & B_{-} \\
\hline B_{+}^{*} X_{\infty}^{\prime} & 0 & 0 & I \\
-C_{+} & -C_{-} & I & 0
\end{array}\right],
$$$$
Z_{\infty}^{\prime}=\left(I-\gamma^{-2} Y_{\infty}^{\prime} X_{\infty}^{\prime}\right)^{-1} \text {, and }
$$

$$
\begin{aligned}
A_{\infty}^{\prime}=A_{+}-\frac{1}{\gamma^{2}-1} Q_{+} R_{+} & -Z_{\infty}^{\prime} Y_{\infty}^{\prime} C_{+}^{*} C_{+} \\
& +\left(\frac{1}{\gamma^{2}-1} Q_{+} Q_{+}^{*}-B_{+} B_{+}^{*}\right) X_{\infty}^{\prime} .
\end{aligned}
$$

Proof: The fact that $\Pi^{\prime}$ still satisfies the assumptions of [9] is trivial to prove and thus omitted.

$$
\begin{gathered}
\text { Now, define } Q=\left[\begin{array}{c}
Q_{+} \\
0
\end{array}\right] \text { and } R=\left[\begin{array}{ll}
R_{+} & 0
\end{array}\right] \text {, then } \\
A-\frac{1}{\gamma^{2}-1} Q R=\left[\begin{array}{cc}
A_{+}-\frac{1}{\gamma^{2}-1} Q_{+} R_{+} & 0 \\
0 & A_{-}
\end{array}\right], \\
R^{*} R=\left[\begin{array}{cc}
R_{+}^{*} R_{+} & 0 \\
0 & 0
\end{array}\right] \text { and } Q Q^{*}=\left[\begin{array}{cc}
Q_{+} Q_{+}^{*} & 0 \\
0 & 0
\end{array}\right] .
\end{gathered}
$$

Therefore, the AREs associated with the Hamiltonians $H_{\infty}$ in (8) and $J_{\infty}$ in (9) have the structure necessary to apply Lemma 1. The proof then follows by applying Theorem 2 to $\Pi^{\prime}$.

It must be noted that, although the above procedure presents a possible significant computational advantage, the resulting controller has the same order as the plant.

It is important to note that such a procedure is not possible when using classical coprime factorizations of the plant ${ }^{2}$. Suppose a plant has a state space realization given by (10) and let $F=\left[\begin{array}{ll}F_{+} & 0\end{array}\right]$ be such that $A_{+}+B_{+} F_{+}$is Hurwitz. Then using the RCF induced by $F$, synthesizing a robustly stabilizing controller requires the solution of the ARE associated with the Hamiltonian matrix [10]

$\tilde{H}_{\infty}=\left[\begin{array}{cc}{\left[\begin{array}{cc}A_{+}-\frac{1}{\gamma^{2}-1} B_{+} F_{+} & 0 \\ -\frac{1}{\gamma^{2}-1} B_{-} F_{+} & A_{-}\end{array}\right]^{*}-\frac{1}{\gamma^{2}-1}\left[\begin{array}{cc}C_{+}^{*} C_{+}-F_{+}^{*} F_{+} C_{+}^{*} C_{-} \\ C_{-}^{*} C_{+} & C_{-}^{*} C_{-}\end{array}\right]} \\ -\frac{\gamma^{2}}{\gamma^{2}-1}\left[\begin{array}{lll}B_{+} B_{+}^{*} & B_{+} B_{-}^{*} \\ B_{-} B_{+}^{*} & B_{-} B_{-}^{*}\end{array}\right] & -\left[\begin{array}{cc}A_{+}-\frac{1}{\gamma^{2}-1} B_{+} F_{+} & 0 \\ -\frac{1}{\gamma^{2}-1} B_{-} F_{+} & A_{-}\end{array}\right]\end{array}\right]$

\footnotetext{
${ }^{2}$ In that case however, one of the two AREs admits the trivial solution and hence in essence the number of states that need to be stabilised in halved.
}

which clearly does not conform to the structure required by Lemma 1 . We now show that this Hamiltonian does not

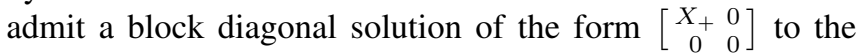
corresponding ARE. Suppose that the ARE associated with the Hamiltonian $\tilde{H}_{\infty}$ has a solution of the form $\left[\begin{array}{cc}X_{+} & 0 \\ 0 & X_{-}\end{array}\right] \geq$ 0 , then

$X_{-} A_{-}+A_{-}^{*} X_{-}-\frac{\gamma^{2}}{\gamma^{2}-1} X_{-} C_{-}^{*} C_{-} X_{-}+\frac{1}{\gamma^{2}-1} B_{-} B_{-}^{*}=0$.

Now if $X_{-}=0$ (as in the BCF case), it follows that $\frac{1}{\gamma^{2}-1} B_{-}^{*} B_{-}=0$ and therefore $B_{-}=0$, which implies that the modes captured by $A_{-}$must be completely uncontrollable for a block diagonal solution to exist.

It would therefore be possible to omit the associated states prior to synthesis, by starting with a minimal state space realisation.

\section{NumericAl EXAMPLE}

A numerical example will be given in this section to demonstrate the applicability of the proposed results. The problem considered is that of a swarm of autonomous vehicles where a centralized controller is tasked with maintaining a formation.

These robots are required to move in a square formation relative to $r_{1}$ as shown Figure 2a. We assume the following hardware setup: $r_{1}$ is equipped with an absolute position measurement system as well as hardware that can be used to measure the relative positions of $r_{2}$ and $r_{3}$. Similarly, $r_{2}$ and $r_{3}$ are able to measure the relative position of $r_{4}$. Finally, $r_{4}$ is not equipped with any position measurement hardware. All robots are assumed to have access to their internal states (acceleration, velocity, etc.). The relative position measurements available in the overall system can be summarized by the directed graph in Figure 2b. Formation control can then be achieved by stabilising the relative position errors according to the same graph.

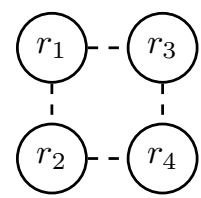

(a) Desired robot formation

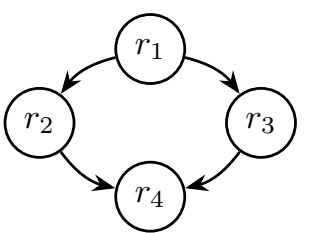

(b) Swarm relative position measurement graph.
For simplicity we consider a swarm of four homogeneous, holonomic omnidirectional ground vehicles, such as the ones considered in [16], these will be denoted henceforth as $r_{i}$ for $i \in\{1, \ldots, 4\}$. Using the local velocity and acceleration measurements available to each robot, linearising velocity controllers are implemented such as the one described in [17], hence each robot can be given a reference velocity which it will track. The transfer matrix mapping the reference velocity to the position of each of these vehicles can be modelled as $P=\frac{1}{s} \hat{P}$ where $\hat{P} \in \mathscr{R} \mathscr{H}_{\infty}^{2 \times 2}$ corresponds to the closed loop velocity dynamics of each robot and has the minimal state space realization $\hat{P}=\left[\begin{array}{l|l}A & B \\ \hline C & 0\end{array}\right]$ with 
$A \in \mathbb{R}^{n \times n}$. Then, noting that $\operatorname{det} A \neq 0$ since $\hat{P} \in \mathscr{R} \mathscr{H}_{\infty}$, a minimal state space realisation of $P$ is given by

$$
P=\left[\begin{array}{cc|c}
0 & C & 0 \\
0 & A & B \\
\hline I & 0 & 0
\end{array}\right]=\left[\begin{array}{cc|c}
0 & 0 & -C A^{-1} B \\
0 & A & B \\
\hline I & C A^{-1} & 0
\end{array}\right] .
$$

Finally, the relative position dynamics can be expressed in terms of a Kronecker product as

$$
Z \otimes P \text { where the Laplacian } Z=\left[\begin{array}{cccc}
1 & -1 & 0 & 0 \\
1 & 0 & -1 & 0 \\
0 & 1 & 0 & -1 \\
0 & 0 & 1 & -1
\end{array}\right] .
$$

Then combining the above with (13) a state space realisation of the overall system can be expressed as

$$
Z \otimes P=\left[\begin{array}{cc|c}
0 & 0 & -I \otimes C A^{-1} B \\
0 & I \otimes A & I \otimes B \\
\hline Z \otimes I & Z \otimes C A^{-1} & 0
\end{array}\right] .
$$

Note that since the Laplacian $Z$ is rank deficient (15) is unobservable. A simple solution to this problem is to redefine $Z$ as

$$
Z=\left[\begin{array}{cccc}
1 & 0 & 0 & 0 \\
1 & -1 & 0 & 0 \\
1 & 0 & -1 & 0 \\
0 & 1 & 0 & -1 \\
0 & 0 & 1 & -1
\end{array}\right]
$$

Since the above has full column rank it follows that the state space realisation given by (15) is observable. Additionally, the inclusion of the position of $r_{1}$ in the output vector leads to a controller covering both the formation of the swarm as well as its absolute position.

We can now proceed to apply the result of Theorem 3 to the system under consideration. First, note that (15) is already in Gilbert form as required by Theorem 3. Now let $Q_{+} \in \mathbb{R}^{2 \times r}$ and $R_{+} \in \mathbb{R}^{r \times 2}$ be such that $Q_{+} R_{+} \in \mathbb{R}^{2 \times 2}$ is Hurwitz which implies via [18, Theorem 13.12] that $I \otimes$ $Q_{+} R_{+}$is also Hurwitz. Finally, define

$G_{+}=\left[\begin{array}{cc|c:c}I \otimes Q_{+} R_{+} & 0 & I \otimes Q_{+} & -I \otimes C A^{-1} B \\ 0 & I \otimes A & 0 & I \otimes B \\ \hdashline I \otimes R_{+} & 0 & I & 0 \\ \hdashline Z \otimes \bar{I}-\bar{Z}_{\bar{C}} \otimes \bar{A}^{-1} & 0 & 0\end{array}\right]$

Then $G_{+} \in \tilde{\mathscr{B}}(Z \otimes P)$.

With the velocity dynamics of each vehicle given by

$$
\hat{P}=\left[\begin{array}{cc|cc}
-2 & 0 & 2 & 0 \\
0 & -3 & 0 & 3 \\
\hline 1 & 0 & 0 & 0 \\
0 & 1 & 0 & 0
\end{array}\right]
$$

and $Q_{+}=-R_{+}=-2 I_{2}$, the Hamiltonians given by Theorem 3 can be constructed as

$$
\begin{gathered}
H_{\infty}^{\prime}=\left[\begin{array}{cc}
4 \epsilon I & (4 \epsilon-1) I \\
-4(1+\epsilon) I & -4 \epsilon I
\end{array}\right] \text { and } \\
J_{\infty}^{\prime}=\left[\begin{array}{cc}
4 \epsilon I & \left(4 \epsilon I-Z^{*} Z\right) \otimes I \\
-4(1+\epsilon) I & -4 \epsilon I
\end{array}\right],
\end{gathered}
$$

where $\epsilon=\left(\gamma^{2}-1\right)^{-1}$. Solving the associated AREs gives

$$
\begin{aligned}
& \operatorname{Ric}\left(H_{\infty}^{\prime}\right)=X_{\infty}^{\prime}=2.359 I_{8} \geq 0 \text { and }
\end{aligned}
$$

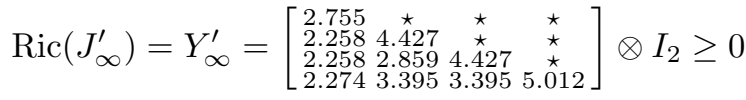

with the spectral radius of their product given by $\rho\left(X_{\infty}^{\prime} Y_{\infty}^{\prime}\right)=5.459$. Finally, a robustly stabilising controller $C_{\infty}^{\prime}$ can be synthesised according to Theorem 3 achieving a robust stability margin $\left\|\mathcal{F}_{l}\left(\Pi_{\infty}^{\prime}, C_{\infty}^{\prime}\right)\right\|_{\infty}=5.466$ against BCF uncertainty of the form depicted in Figure 1. The controller $C_{\infty}^{\prime}$ is given by $C_{\infty}^{\prime}=\left[\begin{array}{c|c}A_{c}^{\prime} & B_{c}^{\prime} \\ \hline C_{c}^{\prime} & 0\end{array}\right]$ where

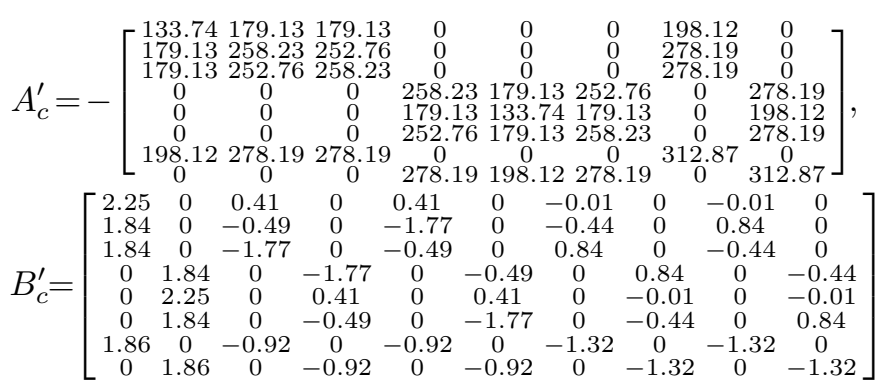

and

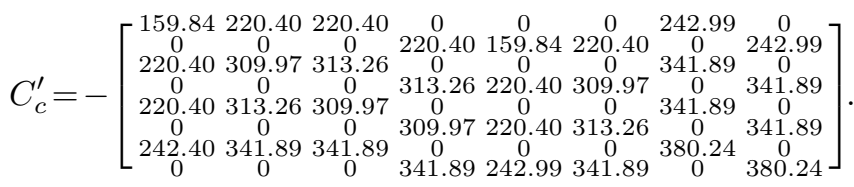

A normalized coprime factor (NCF) controller was also synthesised according to the procedure outlined by [14] and simulated in parallel with the proposed BCF controller for comparison. In both cases no loopshaping weights were used for the synthesis. The simulation results are shown in Figure 3 and Figure 4, depicting the position and relative errors respectively.

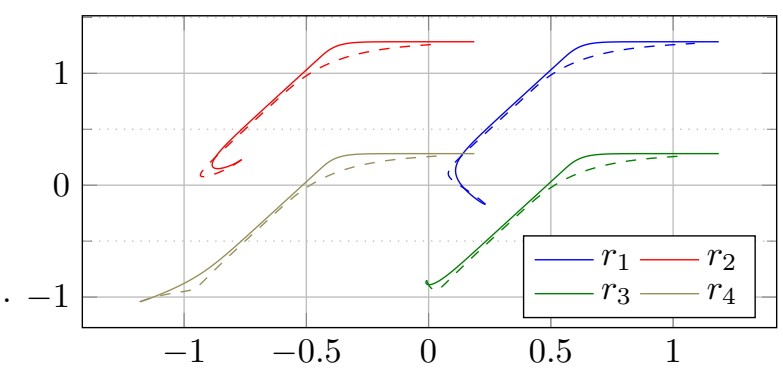

Fig. 3: Robot formation trajectory. Solid and dashed lines correspond to the responses obtained using the $\mathrm{BCF}$ and $\mathrm{NCF}$ controllers respectively.

The advantage of the proposed synthesis method can be observed when considering a dynamically changing number of agents; for example adding new robots to the formation during operation. For every group of new mobile robots added to the network a new controller would need to be synthesised since in this example we consider a centralized control strategy. Suppose now that the total number of robots in the network is changed to $k \geq 1$, then an NCF controller would require the solution of two ${ }^{3}$ ARE with dimension $4 k$ each (since $P$ is $4^{\text {th }}$ order) while the BCF approach would require solving two AREs with dimension $2 k$ each (since $P$

\footnotetext{
${ }^{3}$ Two AREs are required; one to obtain a NCF of the plant using the formulae given by [19] and one to synthesize the controller.
} 


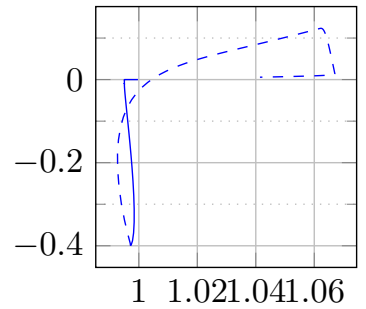

(a) $e_{12}$

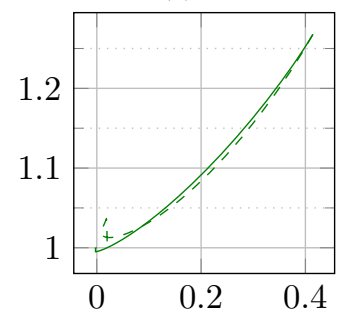

(c) $e_{24}$

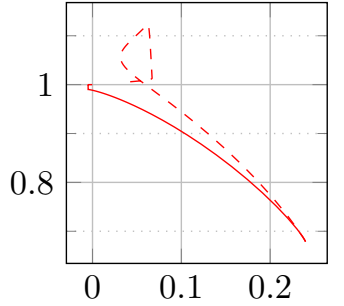

(b) $e_{13}$.

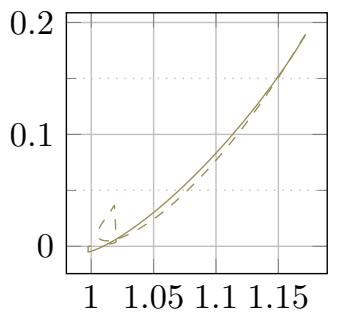

(d) $e_{34}$.
Fig. 4: Relative position error. Solid and dashed lines correspond to the responses obtained using the BCF and NCF controllers respectively.

has two stable well-damped poles that can be discarded and two poles at the origin which must be stabilized). Noting that solving AREs typically has complexity $\mathcal{O}\left(n^{3}\right)$ [20] it follows that the BCF could be constructed significantly faster and thus not affect performance or risk losing stability ${ }^{4}$.

In the presented simple example, the total number of states being stabilised in the two cases is equal which could be misleading; this is a result of the fact that the modes of the agent dynamics are equally split between open left and closed right half complex planes. It is more realistic however that, in practical situations, a plant of the type being considered has more stable modes than unstable ones. Thus the computational advantage afforded by the proposed synthesis method is even more pronounced.

\section{CONCLUSION}

A robust control synthesis theorem has been presented in this paper considering a bicoprime factor uncertainty structure. It was shown that the use of BCFs allows for a separation of stable modes in the plant and thus robust stabilisation can be achieved via the solution of reduced order AREs. This amounts to a possibly significant computational advantage that can be especially beneficial when the controller needs to be dynamically updated such as in gain scheduling scenarios. The presented methodology could be extended using the normalized $\mathrm{BCF}$ results presented in [21] to yield even greater reduction in computational requirements. The theory developed is applied in a numerical example to a swarm of mobile robots in the context of formation control.

\footnotetext{
${ }^{4}$ The imposition of normalization in the NCF example allows for a direct computation of the stabilising controller without the need for iterations that are necessary in the $\mathrm{BCF}$ case. Hence, for a sufficiently small $k$, the NCF approach would be more efficient. This is not the case for large $k$.
}

\section{ACKNOWLEDGMENT}

This work was supported by the Engineering and Physical Sciences Research Council (EPSRC) [grant number EP/R008876/1]. All research data supporting this publication are directly available within this publication.

\section{REFERENCES}

[1] M. Tsiakkas and A. Lanzon, "Bicoprime factor stability criteria and uncertainty characterisation," in Proceedings of the 8th IFAC Symposium on Robust Control Design, Bratislava, Slovakia, Jul. 2015, pp. 228-233.

[2] - "Foundations of a bicoprime factorization theory," IEEE Transactions on Automatic Control, vol. 62, no. 9, pp. 4598-4603, Sept 2017.

[3] H. H. Rosenbrock, "The stability of linear time-dependent control systems," Journal of Electronics and Control, vol. 15, no. 1, pp. 73-80, 1963.

[4] C. Desoer, "Slowly varying system $\dot{x}=a(t) x$," IEEE Transactions on Automatic Control, vol. 14, no. 6, pp. 780-781, Dec 1969.

[5] A. N. Gündeş and C. A. Desoer, Algebraic Theory of Linear Feedback Systems with Full Decentralized Compensators. Berlin: SpringerVerlag, 1990.

[6] K. A. Ünyelioğlu, U. Özgüner, and A. B. Özgüler, "Fixed zeros of decentralized control systems," IEEE Transactions on Automatic Control, vol. 45, no. 1, pp. 146-151, Jan. 2000.

[7] M. Vidyasagar, Control System Synthesis: A Factorization Approach. Morgan \& Claypool Publishers, 2011, reprint.

[8] M. Vidyasagar and H. Kimura, "Robust controllers for uncertain linear multivariable systems," Automatica, vol. 22, no. 1, pp. 85 - 94, 1986.

[9] J. C. Doyle, K. Glover, P. P. Khargonekar, and B. A. Francis, "Statespace solutions to standard $\mathcal{H}_{2}$ and $\mathcal{H}_{\infty}$ control problems," IEEE Transactions on Automatic Control, vol. 34, no. 8, pp. 831-847, Aug. 1989.

[10] K. Zhou, J. C. Doyle, and K. Glover, Robust and Optimal Control. Upper Saddle River, New Jersey: Prentice-Hall, Inc., 1996.

[11] D. McFarlane and G. K., "A loop shaping design procedure using $H_{\infty}$ synthesis," IEEE Transactions on Automatic Control, vol. 37, no. 6, pp. 759-769, Jun. 1992.

[12] A. Lanzon and G. Papageorgiou, "Distance measures for uncertain linear systems: A general theory," IEEE Transactions on Automatic Control, vol. 54, no. 7, pp. 1532-1547, Jul. 2009.

[13] C. N. Nett, C. A. Jacobson, and M. J. Balas, "A connection between state-space and double coprime fractional representations," IEEE Transactions on Automatic Control, vol. 29, pp. 831-832, 1984.

[14] K. Glover and D. McFarlane, "Robust stabilization of normalized coprime factor plant descriptions with $\mathscr{H}_{\infty}$-bounded uncertainty," IEEE Transactions on Automatic Control, vol. 34, no. 8, pp. 821-830, Aug. 1989.

[15] A. Lanzon, S. Engelken, S. Patra, and G. Papageorgiou, "Robust stability and performance analysis for uncertain linear systems - The distance measure approach," International Journal of Robust and Nonlinear Control, vol. 22, no. 11, pp. 1270-1292, Jul. 2012.

[16] F. G. Pin and S. M. Killough, "A new family of omnidirectional and holonomic wheeled platforms for mobile robots," IEEE Trans. Robotics and Automation, vol. 10, pp. 480-489, 1994.

[17] Y. Liu, X. Wu, J. J. Zhu, and J. Lew, "Omni-directional mobile robot controller design by trajectory linearization," in Proceedings of the 2003 American Control Conference, 2003., vol. 4, June 2003, pp. 3423-3428.

[18] A. J. Laub, Matrix Analysis for Scientists \& Engineers. Society for Industrial and Applied Mathematics, 2005.

[19] M. Vidyasagar, "Normalized coprime factorizations for nonstrictly proper systems," IEEE Transactions on Automatic Control, vol. 33, no. 3, pp. 300-301, Mar. 1988.

[20] A. Lanzon, Y. Feng, B. D. O. Anderson, and M. Rotkowitz, "Computing the positive stabilizing solution to Algebraic Riccati Equations with an indefinite quadratic term via a recursive method," IEEE Transactions on Automatic Control, vol. 53, no. 10, pp. 2280-2291, Nov. 2008.

[21] M. Tsiakkas and A. Lanzon, "Normalized bicoprime factorizations," in Proceedings of the 2018 European Control Conference (ECC), Limassol, Cyprus, Jun. 2018, pp. 149-154. 